\title{
Nachruf
}

\section{Wally Kaechele - Eine Kämpferin für die Sache Tanztherapie}

\section{Marianne Eberhard-Kaechele}

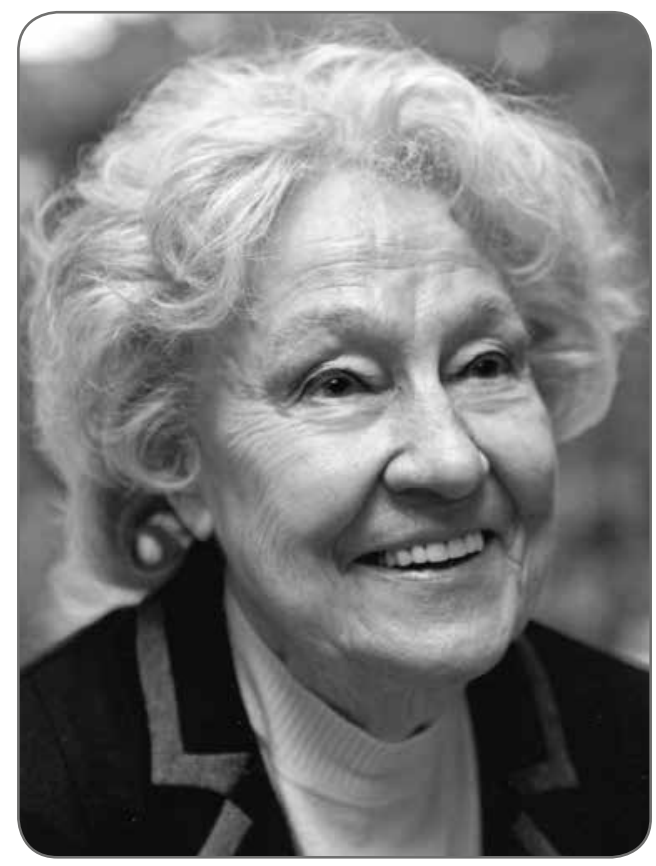

1929 in eine musische Familie hineingeboren, spielte der freie künstlerische Ausdruck für Wally Kaechele schon früh eine Rolle. Noch während sie an der Kunstakademie studierte, entdeckte sie in den 1950er Jahren ihre Leidenschaft für den Tanz. Ihr Ehemann holte sie in seine Gesellschaftstanzschule, die ein breites Spektrum an Tanzformen anbot. Als charismatische und leistungsorientierte Persönlichkeit nahm Wally an Turnieren teil, trat im Showtanz auf und coachte prominente Show- persönlichkeiten wie Gitte Haenning, Paul Kuhn und Howard Carpendale in Haltung und Auftreten. Mit zeitgenössischen Musikern wie Hugo Strasser, Hazy Osterwald und Max Greger nahm sie Schallplatten auf. Doch dies genügte ihr alles nicht.

Sie brach die starren Strukturen des Gesellschaftstanzes auf, indem sie Partytänze erfand, die einzeln oder in Gruppen statt nur paarweise umsetzbar waren und international prämiert wurden. „Fröhliche Bewegungstherapie auf dem Parkett“ sollte in den 196oer Jahren durch Improvisation und Emotionalität die Befreiung aus gesellschaftlichen Zwängen unterstützen und erfuhr eine große Resonanz zunächst von den Teilnehmerlnnen, die ihren Ärzten die positive Wirkung auf körperliche und seelische Leiden schilderten, ähnlich wie bei Marian Chace in den USA.

Ärztliche Fachzeitschriften berichteten dann von ihrer Arbeit, und sie wurde eingeladen auf Ärztekongresse. Die Liste ihrer engen Kontakte zu Ärzten ist lang. Besonders zu erwähnen waren Giesela Eberlein, Vorreiterin der prophylaktischen Nutzung des Autogenen Trainings, Dietrich Langen, erster Lehrstuhlinhaber für Psychotherapie und Medizinische Psychologie in Deutschland, und Wolfgang Jakob von der Universität Heidelberg. Diese Aktivitäten machten das Fernsehen auf Wally aufmerksam. Nach dem Erfolg von Einzelauftritten wurde eine Sendung „Gymnastik einmal anders“ mit ihr produziert, die zunehmend breite 
Bevölkerungsgruppen in Bewegung brachte. In wechselseitiger Verstärkung erregte ihre mediale Präsenz weiter die Aufmerksamkeit der Ärzteschaft und umgekehrt.

Wally lernte in dieser Zeit vier interdependente Faktoren des berufspolitischen Erfolgs verstehen:

1. Die Aktivierung der Betroffenen zur Verdeutlichung ihres Bedarfs

2. Die Sicherung der Unterstützung der Fachwelt durch intensive Kontakte zu Ärzten mit einflussreichen Posten

3. Die Verbündung mit Politikern

4. Die Edukation der Gesellschaft, um die Bekanntheit und Akzeptanz der neuen Methode zu fördern, auch unter Nicht-Betroffenen

Unter TanztherapeutInnen, die diese komplexe Strategie nicht verstanden, wurde Wally dafür angefeindet, zu volkstümlich mit Bewegung zu arbeiten und medial zu populär vorzugehen, wenn sie z.B. Tanztherapie im Fernsehen in Hans Mohls Gesundheitsmagazin Praxis oder in Illustrierten wie der Bunten vorstellte.

Wally unterrichtete alle Altersgruppen sowie verhaltensgestörte Kinder, Umsiedler, Senioren, Gehörlose, Blinde, körperlich und geistig behinderte Menschen und vertrat eine Philosophie, die heute mit Embodimentforschung begründet werden kann: Jeder Mensch kann sich in Bewegung ausdrücken, ganz gleich wie seine körperliche Gestalt ist, weil es um Muster und Metaphern statt um äußere Formen und Schrittfolgen geht. Zutrauen in die eigenen Möglichkeiten und Ressourcenförderung vermittelte sie lange vor der Ressourcenrevolution in der Psychotherapie um die Jahrtausendwende.

Aufgrund ihrer Bekanntheit wurde sie zur ersten international ausgerichteten Tagung der American Dance Therapy Association in Toronto, Kanada, eingeladen. Dort wurde ihr ressourcenorientierter Ansatz positiv aufgenommen, und sie erkannte, wie Tanztherapie eigenständig psychotherapeutisch wirken kann statt nur als Beiwerk zur ärztlichen Psychotherapie. Sie bildete sich fort und arbeitete auf internationalen Kongressen einige Jahre zusammen mit Julianna Lau von der York Universität in Toronto. Im Jahr 1983 gründete sie eine eigene Ausbildungsinstitution nach dem Konzept der York Universität. Entgegen der Annahme ihrer Kritiker kannte Wally ihre fachlichen Grenzen. In der von ihr gegründeten Dietrich Langen Schule (später Langen Institut) unterrichtete sie nur im Bereich heilpädagogischer Tanz. Für den Bereich Tanztherapie engagierte sie jüngere KollegInnen, die in Nord-Amerika eine qualifizierte Ausbildung gemacht hatten, unter anderem Susanne Bender, Marianne Eberhard-Kaechele, Petra Klein, Erika KlettiRanacher, Sybille Scharf-Widder, Sabine Trautmann-Voigt und Bernd Voigt. Diesen und anderen Pionierlnnen der Tanztherapie der zweiten Generation gab Wally Starthilfe für eine eigene Karriere. Sie lud regelmäßig Gastdozenten aus den USA ein, doch mit der Zeit trieb sie die Emanzipation von den amerikanischen Strukturen voran.

Zeitweise führte Wally Kaechele parallel fünf Gesellschaftstanzschulen, das Langen Institut für Tanztherapie und ein Seminar-Hotel auf Ibiza. Diese betriebswirtschaftliche Kompetenz brachte sie in die einzigartige Position, sich in einem Maße für die Berufspolitik zu engagieren wie keiner vor oder nach ihr. Dieses Engagement verstärkte sich, als die ersten AbsolventInnen der Ausbildung Praktikums- und dann Arbeitsplätze suchten. Es entsprach ihrem starken Verantwortungsbewusstsein, jeden, der es wollte, durch ihre Verbindungen und Aktivitäten in eine Anstellung bringen zu wollen.

Wally war zäh und hartnäckig, ein Lazarus, der mehrmals wieder aufstand, nachdem sie oder ein Projekt für tot erklärt wurde. 1993 wurde sie mit Legionellen infiziert und lag monatelang im Koma. Man sagte, sie würde nie wieder laufen oder kommunizieren können, 
doch sie erkämpfte sich den vollen Besitz ihrer Fähigkeiten zurück. Ihr Ziel, eine akademische Ausbildung zu etablieren, verhandelte sie jeweils jahrelang mit der Landesregierung in Düsseldorf, der Folkwang-Hochschule in Essen und der Palucca-Schule in Dresden. Jedes Mal, wenn nach so viel investierter Zeit und Geld ein Projekt wieder kurz vor dem Ziel zu Fall gebracht wurde, schüttelte sich Wally kurz und suchte sofort nach einer neuen Möglichkeit. Verzagen oder Verzweifeln gehörten nicht zu ihrem Repertoire.

Wally war kein Bauer, der Jahr für Jahr das gleiche Feld bestellte. Es war nicht ihre Sache, den täglichen Unterricht oder die Therapie an einem Ort zu machen. Sie war ein Jäger, der immer neue Ziele ins Visier nahm, die viele andere noch gar nicht sahen. Berufspolitische Aktivitäten, die zu einer bestimmten Zeit als neu galten, hatte Wally bereits Jahrzehnte zuvor umgesetzt: Sie hatte eine Deutsche Gesellschaft für Gesundheitsvorsorge, einen Berufsverband der Tanz- und Ausdruckstherapeuten (BdTA), einen Internationalen Verband für Kunst, Gestaltung und Therapien mitgegründet, eine Fachzeitschrift für Tanztherapie herausgegeben, Hochschul-Curricula für die Akademisierung der Tanztherapie erstellt und mit Hochschulen um die Umsetzung verhandelt, ein übergreifendes Berufsbild „Künstlerischer The- rapeut“ erarbeitet, Antworten auf den demografischen Wandel gesucht und vieles mehr. Doch oft verzweifelte sie fast daran, dass zu wenige KollegInnen ihre Voraussicht teilten oder hinterherkamen, um das Territorium, das sie erobert hatte, zu sichern. Und so gerieten ihre Leistungen in Vergessenheit, und das Rad wurde von anderen zu einem späteren Zeitpunkt neu erfunden. Doch sie hielt sich nicht lange mit Groll oder Frustration auf, sondern freute sich über die Sache, wenn ihre Visionen eine Renaissance erfuhren.

Wally Kaechele trug maßgeblich dazu bei, dass Tanztherapie heute in diversen Einrichtungen des Gesundheitswesens stattfindet und in medizinischen Leitlinien und Leistungsverzeichnissen enthalten ist. Dafür erhielt sie von Bundespräsident Rau das Große Bundesverdienstkreuz im Schloss Bellevue und die Würdigung des BTD.

Kurz vor ihrem Tod zeigte sie mir Unterlagen zum Präventionsgesetz und meinte, Tanztherapeutlnnen sollten sich politisch mehr in diese Richtung bewegen. Sie suchte bis zum Schluss die nächste Herausforderung. Am 1. Oktober 2019 ist Wally Kaechele nach längerer Krankheit friedlich aus dem Leben gegangen.

Mit ihren Sieben-Meilen-Stiefeln hat sie die Tanztherapie weit gebracht. Dafür gebühren ihr unser Dank und unsere Anerkennung. 